\title{
EQUIVARIANT BORDISM AND CYCLIC GROUPS
}

\author{
PETER S. LANDWEBER ${ }^{1}$
}

ABSTRACT. For a finite cyclic group $G$ the equivariant complex bordism module $\Omega_{*}^{D}(G)$ is shown to be a free module over $\Omega_{*}^{D}$.

1. Introduction. Let $G$ be a finite group. The equivariant complex bordism module $\Omega_{*}^{U}(G)$ is formed from actions of $G$ on compact stably complex manifolds without restriction on isotropy subgroups. In [5], R. E. Stong shows that $\Omega_{*}^{U}(G)$ is a free module over the complex bordism ring $\Omega_{*}^{U}$ on even-dimensional generators, provided that $G$ is a finite $p$-primary abelian group. One may ask if the same statement holds for other classes of finite groups. In this note we successfully examine this question for finite cyclic groups.

THEOREM 1. If $G$ is a finite cyclic group then $\Omega_{*}^{U}(G)$ is a free $\Omega_{*}^{U}$-module on even-dimensional generators.

At the same time we study the following more general situation. Let $G$ and $H$ be cyclic groups whose orders are relatively prime, and let $B(G, H)$ be a classifying space for $H$-bundles on which $G$ acts as a group of bundle maps (see [4]). Thus $B(G, H)$ is a $G$-space, and the equivariant bordism module $\Omega_{*}^{U}(G)(B(G, H))$ classifies actions of $G \times H$ on compact stably complex manifolds such that $H$ acts freely. Let $H=P_{1} \times \cdots \times P_{r}$ where the $P_{i}$ are the Sylow subgroups of $H$ (cyclic of order $p_{i}^{a_{i}}$ say).

THEOREM 2. (a) $\Omega_{\mathrm{ev}}^{U}(G)(B(G, H)) \cong \Omega_{\mathrm{ev}}^{U}(G)$.

(b) $\Omega_{\text {od }}^{U}(G)(B(G, H)) \cong \oplus_{i=1}^{r} \Omega_{\text {od }}^{U}(G)\left(B\left(G, P_{i}\right)\right)$.

(c) $\Omega_{\mathrm{od}}^{U}(G)\left(B\left(G, P_{i}\right)\right)$ is an $\Omega_{*}^{U}$-module of projective dimension 1 , and consists entirely of $p_{i}$-torsion.

Here $\Omega_{*}^{U}(\quad) \cong \Omega_{\mathrm{ev}}^{U}() \oplus \Omega_{\mathrm{od}}^{U}(\mathrm{)})$ is the decomposition into even and odd components.

The argument we give uses techniques developed by P. E. Conner and E. E. Floyd [2] and R. E. Stong [5] for the analysis of equivariant bordism.

Received by the editors January 11, 1971.

AMS 1969 subject classifications. Primary 5536; Secondary 5710.

Key words and phrases. Equivariant complex bordism, cyclic groups, projective dimension 1.

${ }^{1}$ Research supported in part by the National Science Foundation. 
Mainly we follow the notation of [5]. The next two sections contain preliminaries and a reformulation of the theorems, which are proved in the final section.

2. Families of subgroups. Fix a cyclic group $G$. If $G=K \times L$ we let $\mathscr{F}_{L}=$ the family of all subgroups of the complementary summand $K$. Thus an action of $G$ is $\mathscr{F}_{L}$-free $[5, \S 2]$ if and only if $L$ acts freely, hence

$$
\Omega_{*}^{U}\left(G, \mathscr{F}_{L}\right) \cong \Omega_{*}^{U}(K)(B(K, L)) .
$$

Note that if $L=\{1\}$ then $\mathscr{F}_{\{1\}}=$ all subgroups of $G$ and we recover $\Omega_{*}^{U}(G)$. And if $L=G$ then $\mathscr{F}_{G}=\{\{1\}\}$ and we find the usual isomorphism

for free $G$-actions.

$$
\Omega_{*}^{U}(G,\{\{1\}\}) \cong \Omega_{*}^{U}(B G)
$$

Our plan is to proceed from $\Omega_{*}^{U}(G,\{\{1\}\})$ to $\Omega_{*}^{U}(G)$ through the intermediate stages $\Omega_{*}^{U}\left(G, \mathscr{F}_{L}\right)$. For the induction step we find it necessary to consider bundle bordism modules

$$
\Omega_{*}^{U}\left(G, \mathscr{F}_{L}\right)(B(G, \Gamma))
$$

where $\Gamma$ is a product of unitary groups. Thus we shall really show that the conclusions of Theorems 1 and 2 hold for

$$
\Omega_{*}^{U}(G)(B(G, \Gamma)) \text { and } \Omega_{*}^{U}(G)(B(G, H) \times B(G, \Gamma))
$$

respectively (see $\S 3$ for a more precise statement).

The proof begins by examining the free case, i.e.,

$$
\Omega_{*}^{U}(G,\{\{1\}\})(B(G, \Gamma)) \cong \Omega_{*}^{U}(B G \times B \Gamma) \cong \Omega_{*}^{U}(B G) \otimes_{\Omega_{*}^{U}} \Omega_{*}^{U}(B \Gamma) .
$$

Since $G$ is cyclic, say $G=P_{1} \times \cdots \times P_{r}$ where the $P_{i}$ are its Sylow subgroups: of order $p_{i}^{a_{i}}$, it follows from the bordism spectral sequence that

$$
\Omega_{\mathrm{ev}}^{U}(B G) \cong \Omega_{\mathrm{ev}}^{U} \text { and } \Omega_{\mathrm{od}}^{U}(B G)=\widetilde{\Omega}_{*}^{U}(B G) \cong \bigoplus_{i=1}^{r} \widetilde{\Omega}_{*}^{U}\left(B P_{i}\right)
$$

We know that $\tilde{\Omega}_{*}^{U}\left(B P_{i}\right)$ has projective dimension $1[1, \S 46]$ and consists entirely of $p_{i}$-torsion. Thus $\Omega_{*}^{U}(G,\{\{1\}\})(B(G, \Gamma))$ has the desired form and we have begun the induction.

3. Extensions of actions. Since we are only considering subgroups of the cyclic group $G$ which are summands, extension takes a very simple form. Let

$$
G=K \times L, \quad L=L_{1} \times L_{2}, \quad G_{1}=K \times L_{1} .
$$

Then there is an extension homomorphism

$$
\Omega_{*}^{U}\left(G_{1}, \mathscr{F}_{L_{1}}\right)\left(B\left(G_{1}, \Gamma\right)\right) \stackrel{E}{\longrightarrow} \Omega_{*}^{U}\left(G, \mathscr{F}_{L}\right)(B(G, \Gamma))
$$


which is obtained by simply forming the product with $L_{2}$. That is, if $X \rightarrow M$ is a $\left(G_{1}, \Gamma\right)$-bundle and $L_{1}$ acts freely on $M$, then $X \times L_{2} \rightarrow M \times L_{2}$ is a $(G, \Gamma)$-bundle and $L$ acts freely on $M \times L_{2}$.

LEMMA 3.2. The extension homomorphisms (3.1) are split monomorphisms.

Proof. A left inverse

$$
\Omega_{*}^{U}\left(G, \mathscr{F}_{L}\right)(B(G, \Gamma)) \stackrel{F}{\longrightarrow} \Omega_{*}^{U}\left(G_{1}, \mathscr{F}_{L_{1}}\right)\left(B\left(G_{1}, \Gamma\right)\right)
$$

is obtained by passing to the orbit space of the free $L_{2}$-action. That is, if $X \rightarrow M$ is a $(G, \Gamma)$-bundle and $L$ acts freely on $M$, then $X / L_{2} \rightarrow M / L_{2}$ is a $\left(G_{1}, \Gamma\right)$-bundle and $L_{1}$ acts freely on $M / L_{2}$. It is clear that $F \circ E$ is the identity.

It is convenient to prove the theorems in the following equivalent form. Let $G$ be a finite cyclic group, $G=K \times L$, and let $L=P_{1} \times \cdots \times P_{r}$ where the $P_{i}$ are the Sylow subgroups of $L$. Let $\Gamma$ be a finite product of unitary groups.

THEOREM 1'. $\Omega_{*}^{U}(G)(B(G, \Gamma))$ is a free $\Omega_{*}^{U}$-module on even-dimensional generators.

THEOREM 2'. Extension from $K$ and $K \times P_{i}$ to $G$ induces isomorphisms

and

$$
\Omega_{\mathrm{ev}}^{U}\left(G, \mathscr{F}_{L}\right)(B(G, \Gamma)) \cong \Omega_{\mathrm{ev}}^{U}(K)(B(K, \Gamma))
$$

$$
\Omega_{\mathrm{od}}^{U}\left(G, \widetilde{F}_{L}\right)(B(G, \Gamma)) \cong \bigoplus_{i=1}^{r} \Omega_{\mathrm{od}}^{U}\left(K \times P_{i}, \mathscr{F}_{P_{i}}\right)\left(B\left(K \times P_{i}, \Gamma\right)\right) .
$$

Moreover $\Omega_{\mathrm{od}}^{U}\left(G, \mathscr{F}_{L}\right)(B(G, \Gamma))$ is an $\Omega_{*}^{U}$-module of projective dimension 1 consisting entirely of torsion, and contains p-torsion exactly for those primes dividing the order of $L$.

4. The induction. We now consider the general equivariant bordism module

$$
\Omega_{*}^{U}\left(G, \mathscr{F}_{L}\right)(B(G, \Gamma))
$$

for $L \neq G$. Assume the theorems true if $L$ is replaced by a larger summand of $G$ (if $L=G$ this is the free case treated in $\S 2$; it is easy to check that the isomorphisms are induced by extension), or if $G$ is replaced by a smaller cyclic group. Write

$$
G=K \times P \times L
$$


where $P$ is a Sylow subgroup of $G$. Then we have an exact triangle

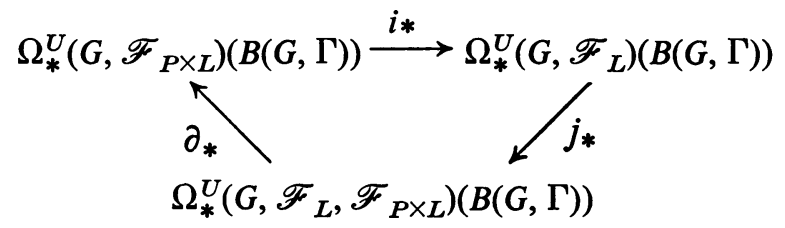

Let $P$ have order $p^{a}$ and let $Z_{p}$ denote its subgroup of order $p$. Then Stong's analysis [5, Proposition 3.4] shows that the relative group is isomorphic to a direct sum

$$
\underset{(j)}{\oplus} \Omega_{*-2|j|}^{U}\left(G / Z_{p}, \mathscr{F}_{L}\right)\left(B\left(G / Z_{p}, \Gamma \times U_{(j)}\right)\right)
$$

where each $U_{(j)}$ is a product of unitary groups $U_{j_{1}} \times \cdots \times U_{j_{r}}$ and $|j|=j_{1}+\cdots+j_{r}$. This arises by passing to the fixed point set of $Z_{p}$.

For brevity we now drop $G$ and $B(G, \Gamma)$ from the notation. By induction $\Omega_{\mathrm{ev}}^{U}\left(\mathscr{F}_{P \times L}\right)$ is a free module over $\Omega_{*}^{U}$ (and so is torsion-free), while $\Omega_{\text {od }}^{U}\left(\mathscr{F}_{L}, \mathscr{F}_{P \times L}\right)$ consists entirely of torsion. Thus

$$
\Omega_{\mathrm{od}}^{U}\left(\mathscr{F}_{L}, \mathscr{F}_{P \times L}\right) \stackrel{\partial_{*}}{\longrightarrow} \Omega_{\mathrm{ev}}^{U}\left(\mathscr{F}_{P \times L}\right)
$$

is zero, hence we obtain a six-term exact sequence

$$
\begin{aligned}
& 0 \longrightarrow \Omega_{\mathrm{ev}}^{U}\left(\mathscr{F}_{P \times L}\right) \stackrel{i_{*}}{\longrightarrow} \Omega_{\mathrm{ev}}^{U}\left(\mathscr{F}_{L}\right) \stackrel{j_{*}}{\longrightarrow} \Omega_{\mathrm{ev}}^{U}\left(\mathscr{F}_{L}, \mathscr{F}_{P \times L}\right) \\
& \stackrel{\partial_{*}}{\longrightarrow} \Omega_{\text {od }}^{U}\left(\mathscr{F}_{P \times L}\right) \stackrel{i_{*}}{\longrightarrow} \Omega_{\text {od }}^{U}\left(\mathscr{F}_{L}\right) \stackrel{j_{*}}{\longrightarrow} \Omega_{\text {od }}^{U}\left(\mathscr{F}_{L}, \mathscr{F}_{P \times L}\right) \longrightarrow 0 .
\end{aligned}
$$

In fact we know more. We have an isomorphism of $\Omega_{\text {od }}^{U}\left(\mathscr{F}_{P \times L}\right)$ with

$$
\Omega_{\mathrm{od}}^{U}\left(K \times P, \mathscr{F}_{P}\right)(B(K \times P, \Gamma)) \oplus \Omega_{\mathrm{od}}^{U}\left(K \times L, \mathscr{F}_{L}\right)(B(K \times L, \Gamma))
$$

(induced by extension from $K \times P$ and $K \times L$ to $G$ ) which we regard as an identification. We proceed to determine $i_{*}$ on these summands; this is the key step.

LeMma 4.2. $i_{*}$ kills $\Omega_{\text {od }}^{U}\left(K \times P, \mathscr{F}_{P}\right)(B(K \times P, \Gamma))$.

Lemma 4.3. The restriction of $i_{*}$ to $\Omega_{\mathrm{od}}^{U}\left(K \times L, \mathscr{F}_{L}\right)(B(K \times L, \Gamma))$ is a split monomorphism.

Proof of Lemma 4.2. We consider the commutative diagram

$$
\begin{array}{cc}
\Omega_{\mathrm{od}}^{U}\left(K \times P, \mathscr{F}_{P}\right)(B(K \times P, \Gamma)) & \stackrel{E}{\longrightarrow} \Omega_{\mathrm{od}}^{U}\left(G, \mathscr{F}_{P \times L}\right)(B(G, \Gamma)) \\
\downarrow^{i_{*}^{\prime}} & \downarrow^{i_{*}} \\
\Omega_{\mathrm{od}}^{U}(K \times P)(B(K \times P, \Gamma)) \stackrel{E^{\prime}}{\longrightarrow} & \Omega_{\text {od }}^{U}\left(G, \mathscr{F}_{L}\right)(B(G, \Gamma))
\end{array}
$$

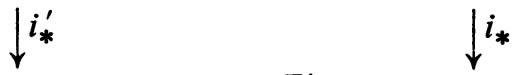


and want to show that $i_{*} E=0$. We claim that $\Omega_{\text {od }}^{U}(K \times P)(B(K \times P, \Gamma))=0$. If $K \times P \neq G$ then this is true by induction, and so we have to consider the case $K \times P=G$ (i.e., $L=\{1\}$ ) further. In this case we extract the exact sequence

$$
\Omega_{\text {od }}^{U}\left(\mathscr{F}^{P}\right) \rightarrow \Omega_{\text {od }}^{U}\left(\mathscr{F}_{\{1\}}\right) \rightarrow \Omega_{\text {od }}^{U}\left(\mathscr{F}_{\{1\}}, \mathscr{F}_{P}\right)
$$

from (4.1) and want to conclude that $\Omega_{\text {od }}^{U}\left(\mathscr{F}_{\{1\}}\right)=0$. Now by induction $\Omega_{\text {od }}^{U}\left(\mathscr{F}_{P}\right)$ consists of $p$-torsion and $\Omega_{\text {od }}^{U}\left(\mathscr{F}_{\{1\}}, \mathscr{F}_{P}\right)=0$. Therefore $\Omega_{\text {od }}^{U}\left(\mathscr{F}_{\{1\}}\right)$ consists of $p$-torsion for each prime which divides $|G|$ (the order of $G$ ), hence $\Omega_{\text {od }}^{U}\left(\mathscr{F}_{\{1\}}\right)=0$ if $|G|$ is not a prime power. On the other hand if $|G|=p^{a}$ so $G=P \cong Z_{p^{a}}$ then it is well known that $\Omega_{\text {od }}^{U}\left(\mathscr{F}_{P}\right) \rightarrow \Omega_{\text {od }}^{U}\left(\mathscr{F}_{\{1\}}\right)$ is zero (the usual generators of the free bordism groups are actions on spheres which bound in the unrestricted bordism group [1]) and so we find again that $\Omega_{\text {od }}^{U}\left(\mathscr{F}_{\{1\}}\right)=0$.

Proof of Lemma 4.3. We must show that the composition

$$
\begin{aligned}
& \Omega_{\text {od }}^{U}\left(K \times K, \mathscr{F}_{L}\right)(B(K \times L, \Gamma)) \stackrel{E}{\longrightarrow} \Omega_{\text {od }}^{U}\left(G, \mathscr{F}_{P \times L}\right)(B(G, \Gamma)) \\
& \stackrel{i_{*}}{\longrightarrow} \Omega_{\text {od }}^{U}\left(G, \mathscr{F}_{L}\right)(B(G, \Gamma))
\end{aligned}
$$

is a split monomorphism. If we follow $i_{*} E$ by the restriction back to $K \times L$ the composition is just multiplication by $|P|$. By induction

$$
\Omega_{\text {od }}^{U}\left(K \times L, \mathscr{F}_{L}\right)(B(K \times L, \Gamma))
$$

is a torsion group on which multiplication by $|P|$ is an isomorphism.

We now observe that (4.1) breaks into two exact sequences

$$
\begin{aligned}
0 & \rightarrow \Omega_{\mathrm{ev}}^{U}\left(\mathscr{F}_{P \times L}\right) \rightarrow \Omega_{\mathrm{ev}}^{U}\left(\mathscr{F}_{L}\right) \rightarrow \Omega_{\mathrm{ev}}^{U}\left(\mathscr{F}_{L}, \mathscr{F}_{P \times L}\right) \\
& \rightarrow \Omega_{\mathrm{od}}^{U}\left(K \times P, \mathscr{F}_{P}\right)(B(K \times P, \Gamma)) \rightarrow 0
\end{aligned}
$$

and

$$
\begin{aligned}
0 & \rightarrow \Omega_{\text {od }}^{U}\left(K \times L, \mathscr{F}_{L}\right)(B(K \times L, \Gamma)) \\
& \rightarrow \Omega_{\text {od }}^{U}\left(\mathscr{F}_{L}\right) \rightarrow \Omega_{\text {od }}^{U}\left(\mathscr{F}_{L}, \mathscr{F}_{P \times L}\right) \rightarrow 0,
\end{aligned}
$$

and the second of these splits. In (4.4) the first and third modules are free and the last one has projective dimension 1 , hence $\Omega_{\mathrm{ev}}^{U}\left(\mathscr{F}_{L}\right)$ is a free module (this argument is due to P. E. Conner and L. Smith [3]). If $L=\{1\}$ we have already seen in the proof of Lemma 4.2 that $\Omega_{\text {od }}^{U}\left(\mathscr{F}_{\{1\}}\right)=0$. If $L \neq\{1\}$ then induction and (4.5) imply that $\Omega_{\text {od }}^{U}\left(\mathscr{F}_{L}\right)$ has projective dimension 1 and consists entirely of torsion, admitting $q$-torsion if and only if the prime $q$ divides $|L|$.

It remains to establish the isomorphisms of Theorem $2^{\prime}$. Consider the 
commutative diagram with exact rows

$$
\begin{aligned}
& 0 \rightarrow \Omega_{\mathrm{ev}}^{U}\left(K \times P, \mathscr{F}_{P}\right) \rightarrow \Omega_{\mathrm{ev}}^{U}\left(K \times P, \mathscr{F}_{\{1\}}\right) \rightarrow \Omega_{\mathrm{ev}}^{U}\left(K \times P, \mathscr{F}_{\{1\}}, \mathscr{F}_{P}\right) \\
& \downarrow \\
& 0 \rightarrow \Omega_{\mathrm{ev}}^{U}\left(G, \mathscr{F}_{P \times L}\right) \rightarrow \Omega_{\mathrm{ev}}^{U}\left(G, \mathscr{F}_{L}\right) \quad \rightarrow \quad \Omega_{\mathrm{ev}}^{U}\left(G, \mathscr{F}_{L}, \mathscr{F}_{P \times L}\right) \\
& \rightarrow \Omega_{\text {od }}^{U}\left(K \times P, \mathscr{F}_{P}\right) \rightarrow 0 \\
& \rightarrow \Omega_{\text {od }}^{U}\left(K \times P, \mathscr{F}_{P}\right) \rightarrow 0
\end{aligned}
$$

obtained from (4.4) by extension from $K \times P$ to $G$. By induction the first and third vertical maps are isomorphisms, hence the five lemma implies that also

$$
\Omega_{\mathrm{ev}}^{U}(K \times P)(B(K \times P, \Gamma)) \stackrel{E}{\longrightarrow} \Omega_{\mathrm{ev}}^{U}\left(G, \mathscr{F}_{L}\right)(B(G, \Gamma))
$$

is an isomorphism.

Next let $L=P_{1} \times \cdots \times P_{r}$ where the $P_{i}$ are the Sylow subgroups of $L$. By induction we have isomorphisms

$$
\Omega_{\mathrm{od}}^{U}\left(K \times L, \mathscr{F}_{L}\right)(B(K \times L, \Gamma)) \cong \bigoplus_{i=1}^{r} \Omega_{\mathrm{od}}^{U}\left(K \times P_{i}, \mathscr{F}_{P_{i}}\right)\left(B\left(K \times P_{i}, \Gamma\right)\right)
$$

and

$$
\begin{aligned}
\Omega_{\mathrm{od}}^{U}\left(\mathscr{F}_{L}, \mathscr{F}_{P \times L}\right) & \cong \underset{(j)}{\bigoplus} \Omega_{\mathrm{od}-2|j|}^{U}\left(G / Z_{p}, \mathscr{F}_{L}\right)\left(B\left(G / Z_{p}, \Gamma \times U_{(j)}\right)\right) \\
& \cong \underset{(j)}{\bigoplus} \underset{i=1}{r} \Omega_{\mathrm{od}-2|j|}^{U}\left(G_{i} / Z_{p}, \mathscr{F}_{L}\right)\left(B\left(G_{i} / Z_{p}, \Gamma \times U_{(j)}\right)\right) \\
& \cong \underset{i=1}{\oplus} \Omega_{\mathrm{od}}^{U}\left(G_{i}, \mathscr{F}_{P \times P_{i}}, \mathscr{F}_{P_{i}}\right)
\end{aligned}
$$

where we have put $G_{i}=K \times P \times P_{i}$. Thus (4.5) yields a commutative diagram with split exact rows

$$
\begin{aligned}
& 0 \rightarrow \underset{i}{\oplus} \Omega_{\mathrm{od}}^{U}\left(K \times P_{i}, \mathscr{F}_{P_{i}}\right) \rightarrow \oplus_{i} \Omega_{\mathrm{od}}^{U}\left(G_{i}, \mathscr{F}_{P_{i}}\right) \\
& 0 \rightarrow \Omega_{\text {od }}^{U}\left(K \times L, \mathscr{F}_{L}\right) \quad \rightarrow \Omega_{\text {od }}^{U}\left(G, \mathscr{F}_{L}\right) \\
& \rightarrow \oplus \Omega_{\text {od }}^{U}\left(G_{i}, \mathscr{F}_{P_{i}}, \mathscr{F}_{P \times P_{i}}\right) \rightarrow 0 \\
& \rightarrow \quad \Omega_{\text {od }}^{U}\left(G, \mathscr{F}_{L}^{\downarrow}, \mathscr{F}_{P \times L}\right) \rightarrow 0
\end{aligned}
$$

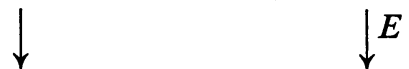

for brevity we have omitted the various classifying spaces from the 
notation). Thus by the five lemma we obtain an isomorphism

$$
\underset{i}{\oplus} \Omega_{\text {od }}^{U}\left(G_{i}, \mathscr{F}_{P_{i}}\right)\left(B\left(G_{i}, \Gamma\right)\right) \stackrel{E}{\longrightarrow} \Omega_{\text {od }}^{U}\left(G, \mathscr{F}_{L}\right)(B(G, \Gamma)) .
$$

This completes the induction and so both theorems are proved.

\section{REFERENCES}

1. P. E. Conner and E. E. Floyd, Differentiable periodic maps, Ergebnisse der Mathematik und ihrer Grenzgebiete, N. F., Band 33, Academic Press, New York; SpringerVerlag, Berlin, 1964. MR 31 \#750.

2. - Maps of odd period, Ann. of Math. (2) 84 (1966), 132-156. MR 34 \#3587.

3. P. E. Conner and L. Smith, On the complex bordism of finite complexes, Inst. Hautes Études Sci. Publ. Math. No. 37 (1969), 117-221. MR 42 \#2473.

4. T. tom Dieck, Faserbündel mit Gruppenoperation, Arch. Math. (Basel) 20 (1969), 136-143. MR 39 \#6340.

5. R. E. Stong, Complex and oriented equivariant bordism, Topology of Manifolds, edited by J. C. Cantrell and C. H. Edwards, Jr., Markham, Chicago, Ill., 1970, pp. 291-316.

Department of Mathematics, Rutgers University, The State University of New Jersey, New Brunswick, New Jersey 08903 